\title{
Eventos adversos após vacinação contra influenza em idosos, Distrito de Campinas, SP, 2000
}

\author{
Adverse reactions to influenza vaccine in the elderly, \\ Campinas District, SP, 2000
}

\author{
Maria Rita Donalisio', Raquel Maria Ramalheira² e Ricardo Cordeiro³
}

\begin{abstract}
Resumo Há poucos dados sistematizados sobre eventos adversos da vacina contra influenza no Brasil. Este trabalho visou identificar estes eventos em população acima de 60 anos que compareceu à Campanha Nacional de Vacinação do Idoso, em Distrito de Campinas, SP, em 2000. Foi realizada entrevista para relato de sintomas gerais e locais, com nexo temporal após a aplicação do imunobiológico, em amostra aleatória sistemática da população $(n=206)$. Registraram-se 20,38\% (IC 14,87-25,88) dos indivíduos com um ou mais sintomas, sendo a dor no local da vacina, a mais freqüente 12,6\% (IC 8,09-17,15). Ajustou-se um modelo de regressão logística múltipla, tendo como variável dependente, a ocorrência de pelo menos um evento adverso. A variável independente que se mostrou associada às reações adversas foi o sexo (feminino) (OR=5,89 e IC 2,08-16,68). Os achados deste estudo reafirmam a pequena reatogenicidade da vacina contra a influenza.
\end{abstract}

Palavras-chaves: Evento adverso. Vacinação. Influenza. Idoso.

Abstract Adverse reactions associated with vaccines are poorly documented in Brazil. The aim of this article was to identify the incidence of adverse effects to the influenza vaccine in the elderly of Campinas district, SP. A questionnaire was applied to a random systematic sample of people over 60 years of age $(\mathrm{n}=206)$, participating in the Brazilian Vaccination Campaign during 2000. Temporal link was taken into account for general and local symptoms after vaccine. One or more symptoms were reported by $20.48 \%$ (Cl 14.87-25.88) and local pain was the most frequent complaint $12.62 \%$ (Cl 8.09-17.15). A multi-regression logistic model was adjusted, considering any adverse effects after exposure as the dependent variable. The independent variable associated with adverse effects was female gender ( $\mathrm{OR}=5.89$ and $\mathrm{Cl}$ 2.08-16.68). This study reaffirms the low reactogenicity of influenza vaccine.

Key-words: Adverse Effect. Vaccination. Influenza. Elderly.

Muitos estudos têm demonstrado o impacto da vacinação contra a influenza na prevenção de internações e mortes por pneumonias e outras doenças, tanto em idosos saudáveis como em populações de risco, particularmente em períodos de maior circulação do vírus ${ }^{59}$. Vale ressaltar que embora a resposta imunológica à vacina contra influenza seja mais discreta em idades mais avançadas, os reais benefícios da vacinação de idosos estão na capacidade de prevenir quadros de pneumonia viral primária e bacteriana secundária (50 a 60\%), internações $(50 \%)$ e reduzir a mortalidade $(80 \%)$. No adulto jovem, a eficácia da vacina atinge 70 a $90 \%$ enquanto que em maiores de 60 anos cai para $40 \%$. A eficácia da vacina depende também da coincidência da composição do imunobiológico com as cepas circulantes na estação ${ }^{114}$.

No Brasil, a vacinação antigripal está disponibilizada em âmbito nacional na campanha de vacinação do idoso desde $1999^{8}$. No estado de São Paulo, a primeira campanha ocorreu em 1998, atingindo cobertura de $70 \%$ em maiores de 60 anos. Em 1999, Ano Internacional do Idoso, chegou a $87,3 \%$ e no ano 2000 a cobertura baixou para $63 \%$ na faixa de 65 anos e mais no Estado ${ }^{14}$.

\footnotetext{
1. Departamento de Medicina Preventiva e Social da Faculdade de Ciências Médicas da Universidade Estadual de Campinas, Campinas, SP. 2. Vigilância Epidemiológica/DIR-XII- Secretaria Estadual de Saúde São Paulo, São Paulo, SP. 3. Departamento de Saúde Pública da Faculdade de Medicina de Botucatu da Universidade Estadual Paulista, Botucatu, SP.

Endereço para correspondência: Profa Maria Rita Donalisio Cordeiro. Caixa Postal 543, 18618-970 Botucatu SP.

Tel:14 6824-8279 Fax:14 6822-3309

e-mail: rita@fmb.unesp.br

Recebido para publicação em 7/10/2002

Aceito em 12/6/2003
} 
Em Campinas, Estado de São Paulo (1 milhão de habitantes), em 1998, foram vacinados $43 \%$ dos idosos; 73,2\% em 1999 e no ano 2000, 64\%. O município ficou entre os que não atingiram a meta mínima de cobertura de $70 \%{ }^{14}$. O Distrito de Barão Geraldo localiza-se na região norte da cidade e possui população de 29.951 habitantes, com 2.144 indivíduos na faixa etária de maiores de 60 anos. A região possui um centro de saúde de fácil acesso à comunidade local, onde foi realizada a pesquisa. As coberturas vacinais no distrito foram semelhantes às do município nos anos de 1998 a 2000.

Ainda existem dificuldades na ampliação da cobertura nesta faixa etária, embora a vacina contra o vírus da influenza esteja disponível e os eventos adversos notificados sejam leves e sem importância clínica ou epidemiológica 671516 .

No Brasil, há poucos trabalhos publicados que apresentam dados a respeito de reações adversas da vacina contra a influenza ${ }^{15}{ }^{16}$. E freqüente a subnotificação e a falta de sistematização destes dados pelo Sistema de Vigilância Epidemiológica.

Este artigo procura identificar a ocorrência de eventos adversos da vacina contra influenza, após a Campanha Nacional de Vacinação do Idoso, em um Distrito do município de Campinas no ano 2000, bem como analisar variáveis possivelmente relacionadas às queixas pós-vacinais na população alvo da campanha.

\section{MATERIAL E MÉTODOS}

Investigou-se a ocorrência de sintomas potencialmente relacionados à vacina em amostra da população acima de 60 anos de idade, que compareceu à vacinação contra o vírus da influenza (735 indivíduos), no Centro de Saúde do Distrito de Barão Geraldo, Campinas, na Semana de Vacinação do Idoso, em abril de 2000.

Durante o comparecimento à vacinação, os indivíduos foram informados sobre os objetivos da pesquisa, obtendose o seu consentimento para participação na investigação, isto é, posterior visita domiciliar e entrevista.

Estabelecendo-se um erro amostral $\delta=0,05$, um valor $z$ igual a 1,96 relativo a um intervalo de confiança de 0,95 (a bilateral de 0,025 ), e pressupondo-se uma proporção amostral de eventos esperados igual a 0,2, dentro de um universo de 735 idosos vacinados, o tamanho amostral ${ }^{2}$ foi calculado como $n=184$, ao qual foi acrescido $10 \%$ para compensar perdas, chegando-se a $\mathrm{N}=203$.

A partir de sorteio de um entre os 3 primeiros indivíduos do cadastro de comparecimento à vacinação, os demais indivíduos foram sistematicamente alocados com passo amostral ${ }^{2}$ igual a 3 .

Foram aplicados 206 questionários compostos por perguntas abertas e objetivas. Foram considerados os sintomas referidos a partir do $5^{\circ}$ dia e no máximo até o $15^{\circ}$ dia após a vacinação, período limitado para facilitar a lembrança de sintomas após a vacina. As variáveis coletadas foram: idade, sexo, ocupação, trabalho atual, antecedentes mórbidos, sintomas nos últimos 15 dias, vínculo e procura por serviços de saúde (conveniados e/ ou particulares ou públicos). No final da entrevista, os indivíduos foram questionados ativamente sobre: febre, mal estar, dor muscular, dor no local da vacina, e sintomas respiratórios. Da mesma forma, perguntou-se sobre o tempo de aparecimento e de duração destes sintomas. A identificação de eventos adversos graves e raros como, por exemplo, a síndrome de Guillan-Barré (1 caso para 1 milhão de vacinados) bem como quadros alérgicos ${ }^{1}$ não foram previstos no cálculo do tamanho da amostra.

Foram considerados eventos adversos, as queixas referidas no período de no máximo 2 dias a partir da vacinação e com duração máxima de 2 dias, conforme indica a literatura ${ }^{14}$. No caso de dor no local da aplicação da vacina, somente o tempo de aparecimento foi considerado.

Os critérios de inclusão no estudo foram ter mais de 60 anos, residir no Distrito de Barão Geraldo, aceitar ser entrevistado e ter tomado somente a vacina contra a influenza, embora estivessem sendo aplicadas também a anti-pneumocócica aos indivíduos que apresentassem solicitação médica por escrito e a vacina dupla adulto em indivíduos com a última dose há mais de 10 anos. Dentre os 735 idosos que compareceram à vacinação, $72,7 \%$ (534) receberam somente a contra a influenza. Optou-se por incluir os indivíduos expostos unicamente a esta vacina, embora este critério possa ter selecionado maior percentual de sadios, devido aos critérios de indicação da anti-pneumocócica ${ }^{14}$.

A vacina utilizada na Campanha foi a Vaxigripe ${ }^{\circledR}$, composta por vírus inativados, purificados, obtidos a partir de células de ovo de galinha, de acordo com as recomendações da Organização Mundial da Saúde para 2000, cepas análogas a: A/Moscow/10/99 ou A/Sydney/ 5/97 (H3N2); A/New Caledonia/20/99 (H1N1); B/Beijing/ 184/93. Estas cepas possuem características antigênicas semelhantes à dos vírus circulantes no ano anterior $^{1416}$. A vacina foi aplicada por via intramuscular em região deltóide com dose correspondente a $0,5 \mathrm{ml}$, sendo $100 \%$ pertencentes ao lote $4057 / \mathrm{A}^{14}$.

As profissões referidas pelos indivíduos foram classificadas de acordo com a Classificação de Rumel $(1987)^{13}$

A partir dos dados colhidos, estimou-se a proporção de incidência ${ }^{11}$ dos efeitos adversos após a exposição à vacina contra a influenza na população que procurou vacinação em 2000, naquela região da cidade.

Ajustou-se um modelo de regressão logística múltipla, tendo como variável dependente, dicotômica $(0=$ não, $1=\operatorname{sim}$ ), a ocorrência de pelo menos um dos seguintes eventos adversos após a vacinação: dor no local da 
vacinação, febre, mal estar, dor muscular e sintomas respiratórios. Como variáveis independentes, o modelo utilizou sexo, idade, antecedentes mórbidos, grupos de ocupação, vínculo a serviços de saúde públicos ou conveniados/privados. Foram excluídos do modelo final os antecedentes mórbidos e ocupações, cuja associação com eventos adversos, na análise univariada, obteve $p>0,30$.

Os cálculos foram feitos utilizando-se o Programa Estatístico SAS Logistic Procedure. O programa EPIINFO versão 6.04 b foi empregado para a construção do banco de dados.

\section{RESULTADOS}

A população estudada (206 indivíduos) foi composta de $56,8 \%$ de mulheres e $43,2 \%$ de homens. A média de idade foi de 70,2 e a mediana de 69 anos. A Tabela 1 mostra a distribuição etária dos indivíduos investigados. A proporção de incidência de eventos adversos à vacina contra influenza não variou de forma significativa entre as faixas etárias $(p=0,59$ quiquadrado=2,78 $\mathrm{gl}=4$ ) (Tabela 1 ).

Tabela 1- Proporção de incidência de sintoma pós-vacinal* (por 100 vacinados) por faixa etária. Distrito Barão Geraldo Campinas 100 vacinad
$S P, 2000$.

\begin{tabular}{lccc}
\hline Faixa etária & Incidência & I. Confiança 95\% & $\mathrm{n}^{\circ}$ \\
\hline $60-64$ & 14,00 & $4,4-23,84$ & 50 \\
$65-69$ & 20,34 & $10,07-30,61$ & 59 \\
$70-74$ & 21,15 & $10,05-32,24$ & 52 \\
$75-79$ & 30,44 & $11,63-49,24$ & 23 \\
$\geq 80$ & 22,73 & $5,22-40,24$ & 22 \\
\hline
\end{tabular}

Chi-quadrado $=2,78 \mathrm{gl}=4$ e $\mathrm{p}=0,59$

*Relato de pelo menos um dos sintomas pós-vacinais: febre, mal estar, cefaléia, dor no local da vacina, respiratórios, outros.

A Tabela 2 mostra a distribuição dos eventos adversos entre os vacinados, evidenciando a dor no local da injeção como o evento de maior freqüência $12,62 \%$ (IC 8,09-17,15). Como outros sintomas foram registrados 1 caso de prurido, 3 casos de cefaléia e 1 de dor de garganta e ouvido.

Tabela 2 - Proporção de incidência de eventos adversos (por 100 vacinados) após a vacina contra influenza, em idosos em Distrito de Campinas SP - 2000.

\begin{tabular}{lccr}
\hline Sintomas referidos & Incidência & I. Confiança $95 \%$ & Casos \\
\hline Febre & 1,94 & $0,98-2,90$ & 4 \\
Mal estar & 7,28 & $5,47-9,09$ & 15 \\
Dor muscular & 1,94 & $0,98-2,90$ & 4 \\
Sintomas gerais * & 7,76 & $4,11-11,41$ & 16 \\
Dor no local da vacina & 12,62 & $8,09-17,15$ & 26 \\
Respiratórios & 2,91 & $1,74-4,08$ & 6 \\
Outros & 2,43 & $0,33-4,53$ & 5 \\
\hline Qualquer sintoma** & 20,38 & $14,87-25,88$ & 42
\end{tabular}

* Pelo um sintoma geral como febre e/ou dor muscular/no corpo e/ou mal estar.

** Indivíduos que referiram pelo menos um evento adverso após a vacina $(\mathrm{N}=206)$. Mais de um sintoma freqüentemente foram referidos pelos indivíduos.

Dos 42 indivíduos que referiram pelo menos 1 sintoma, apenas 2 disseram ter procurado o hospital, ambos com febre e mal estar, 1 procurou a farmácia devido a mal estar e dor no corpo, 18 responderam terem utilizado medicação caseira, sintomáticos e repouso e o restante disse não ter feito nada. Vale ressaltar que não foram notificadas reações adversas graves à vacinação contra influenza pelo Sistema de Vigilância Epidemiológica, no período.

A variável que descreve o uso e vínculo a serviços de saúde mostrou que 93 (45,2\%) indivíduos relataram procurar serviços conveniados e/ou particulares. Em $80(38,8 \%)$ entrevistas houve relato de utilização de serviços públicos (hospitais e/ou centro de saúde) e $33(16 \%)$ não responderam a questão.

Entre os 206 indivíduos entrevistados, 123 (62,1\%) (IC 55,47-68,72), responderam serem indivíduos aposentados, e entre eles 35 (28,5\%) não referiram suas profissões. A Tabela 3 apresenta as proporções de incidência de pelo menos uma queixa compatível com evento adverso pós-vacinal, por 100 vacinados entre os grupos de profissões. Não houve diferença significativa entre os grupos (chi-quadrado 5,47 $\mathrm{gl}=6$ e $\mathrm{p}=0,49$ ).

A Tabela 4 mostra estatísticas obtidas no modelo de regressão logística apontando a associação da variável sexo com a ocorrência de eventos adversos. Observase nesta tabela que as variáveis: idade, ocupação e vínculo a serviços de saúde (conveniado/particular) não se apresentaram significantes no modelo $(p<0,05)$.

Tabela 3 - Proporção de incidência de sintoma pós-vacinal (por 100 vacinados) por grupos de profissões. Distrito Barão Geraldo Campinas SP, 2000.

\begin{tabular}{lccr}
\hline Grupo de profissões* & Incidência & I. Confiança 95\% & N $^{\circ}$ \\
\hline Do lar & 26,08 & $15,74-36,46$ & 69 \\
Agricultores & 7,14 & $6,35-20,55$ & 14 \\
Serviços & 19,04 & $7,14-30,86$ & 42 \\
Intelectuais & 11,76 & $3,54-27,14$ & 17 \\
Operários & 10,52 & $3,28-24,28$ & 19 \\
Autônomo & 33,33 & $0-86,63$ & 3 \\
Sem informação & 23,81 & $17,23-30,37$ & 42 \\
\hline Total & 20,38 & $14,87-25,88$ & 206
\end{tabular}

Chi-quadrado $=5,47 \mathrm{gl}=6$ e p=0,49. * Classificação de Rumel

Tabela 4 - Fatores de risco obtidos pela regressão logística, para ocorrência de eventos adversos da vacinação contra influenza em idosos, Distrito de Campinas SP, 2000.

\begin{tabular}{lrcrc}
\hline Variáveis & $\beta$ & $\mathrm{p}$ & $\mathrm{OR}$ & I. Confiança 95\% \\
\hline Sexo & 1,77 & 0,0008 & 5,89 & $2,08-16,68$ \\
Idade & 0,01 & 0,63 & 1,01 & $0,96-1,07$ \\
Convênio/particular* & 0,46 & 0,25 & 1,58 & $0,73-3,44$ \\
Do lar** $_{\text {Agricultor** }}^{*}$ & $-0,51$ & 0,26 & 0,59 & $0,25-1,46$ \\
\hline OR-Odds Ration * & $-1,45$ & 0,19 & 0,23 & $0,02-2,07$ \\
\hline
\end{tabular}

OR-Odds Ratio. * Serviços de saúde que procura-conveniado ou médico particular. ${ }^{*}$ Grupo da Ocupação - que exerce ou exerceu antes da aposentadoria segundo classificação de Rumel. 


\section{DISCUSSÃO}

Embora a população estudada apresente algumas características semelhantes à população idosa da cidade e região, quanto ao sexo e cobertura vacinal, a generalização dos resultados deste estudo devem ser vistos com cautela, pois trata-se de uma população de usuários de um serviço de saúde e não de uma amostra aleatória de base populacional. Todavia, o objetivo deste estudo focalizou a análise dos eventos adversos relacionados ao imunobiológico específico e procurou compará-los com os observados por outros autores.

Optou-se por não eleger grupo controle com placebo, para não perder a oportunidade de vacinação a qualquer indivíduo que comparecesse ao serviço de saúde. Desta forma, a incidência dos eventos adversos nesta população baseou-se em relato de sintomas após vacinação, inclusive de febre. A falta de um grupo controle não permitiu inferir com rigor, os sintomas que realmente puderam ser atribuídos à vacina.

Foram consideradas perdas somente indivíduos amostrados que não puderam ser contatados. Estas foram menores de 3\%, provavelmente devido à facilidade de se encontrar o idoso em casa, comparada ao adulto em idade produtiva.

Este estudo sugere que a vacinação é pouco reatogênica, corroborando com resultados de outros autores ${ }^{4} 671516$. Embora tenha sido observado $20,4 \%$ de indivíduos com pelo menos um sintoma, estes foram leves, não implicando em procura de serviços de saúde, em mais de $98 \%$ dos casos. A dor no local da aplicação foi a queixa mais freqüente, principalmente em mulheres, como também mostram outros autores ${ }^{4} 6$ (Tabela 1).

Ensaios clínicos duplo-cego encontraram relato de sintomas sistêmicos na mesma proporção, em indivíduos que receberam vacina e placebo ${ }^{6}{ }^{7}$. Margolis et $\mathrm{al}^{7}$ notificaram $27,7 \%$ de sintomas após vacinação, em idosos, sem diferença com o grupo placebo. Tem-se atribuído à vacina, $1 \%$ de sintomas inespecíficos como febre e mal estar, referidos após a imunização ${ }^{14}$.

O relato de sintomas respiratórios tem sido notificado como coincidência no período pós-vacinal, sem diferença entre os grupos de vacinados e placebo ${ }^{7}$. Embora na população do Distrito a proporção de incidência de sintomas respiratórios tenha sido de 2,9 por 100 vacinados, esta ocorrência pode influenciar negativamente a predisposição de receber novas doses de vacina, por parte de idosos.

Preocupação com a ocorrência de eventos adversos tem sido apontada como um fator que contribui para as baixas coberturas vacinais ${ }^{312}$. Muitas vezes, atribuem-se à imunização, sintomas nem sempre associados verdadeiramente à vacina. Estudos sobre a percepção e representação dos sintomas após vacinação podem esclarecer parte dos motivos de baixas coberturas vacinais e contribuir para intervenções educativas mais específicas.

Ao selecionar idosos que só tomaram a vacina contra a influenza, analisou-se uma população que, possivelmente apresenta menor prevalência de doenças crônicas, comparada à população total, pois doenças cardiovasculares, pulmonares, metabólicas indicaram formalmente a utilização da vacina antipneumocócica, em idosos na Campanha6 141516 . Desta forma, a incidência de eventos adversos referidos pode não ser comparável a de estudos que analisam a totalidade da população idosa ${ }^{6}$.

$\mathrm{Na}$ análise multivariada, o sexo (feminino) apresentouse associado ao relato de sintomas pós-vacinais, como encontrado por outros autores ${ }^{467}$. As mulheres mostraramse mais propensas a referirem quaisquer dos sintomas questionados, particularmente a dor no local da injeção, mesmo após ajuste de variáveis como idade e profissão, na análise multivariada (Tabela 4). Dor no local da vacina pode estar associada com a quantidade de tecido gorduroso e espessura da região deltóide, desviando o conteúdo da injeção ao tecido subcutâneo, de maior enervação. Este fenômeno é mais comum em mulheres ${ }^{10}$.

Embora os antecedentes mórbidos hipertensão e diabetes sejam de grande prevalência nesta faixa etária, estas variáveis não apresentaram associação significativa com a ocorrência de qualquer evento adverso na análise univariada ( $p>0,30$ ), tampouco na multivariada.

Quanto aos grupos de ocupação, nenhum deles foi associado ao relato de sintomas adversos, nem mesmo com o fato de não trabalhar e de ser aposentado.

O vínculo a convênio de saúde e procura de serviços particulares em contraposição aos serviços públicos (centro de saúde e hospital) podem significar acesso diferenciado a serviços de saúde, vínculo empregatício no presente ou clientela com maior poder aquisitivo. Mesmo assim, no modelo logístico, esta variável não se mostrou associada ao relato de queixas pós-vacinais.

É importante lembrar as limitações das associações estatísticas de modelos multivariados, que eventualmente podem não refletir verdadeiras associações causais, exigindo investigação mais detalhada.

Este estudo reforça a importância da notificação e vigilância epidemiológica para esclarecimento oportuno de eventos adversos, mesmo de vacinas pouco reatogênicas como a influenza.

\section{AGRADECIMENTOS}

Aos residentes do Departamento de Medicina Preventiva e Social da Faculdade de Ciências Médicas da Universidade Estadual de Campinas, Carlos Alberto Henn, Bernadete Perez Coelho e Danielle Ribeiro Moraes, pela ajuda no campo. 


\section{REFERÊNCIAS BIBLIOGRÁFICAS}

1. Centers for Disease Control and Prevention (CDC). Prevention and Control of influenza: recommendations of the Advisory Committee on Immunization Practices (ACIP). Morbidity Mortality Weekly Report 49(RR03): 1-38, 2000.

2. Cochran WG. Sampling Techniques. Third edition, John Wiley \& Sons, New York, 1977.

3. Fierbach NH, Viscoli CM. Patient acceptance of influenza vaccination: knowledge, attitudes and behavior among high risk outpatients. Archives of Internal Medicine 152:106-110, 1992.

4. Govaert THME, Dinant GJ, Aretz K, Masurel N, Sprenger MJW, Knottnerus JA. Adverse reactions to influenza vaccine in elderly people: randomized double blind placebo controlled trial. British Medical Journal 307:988-990, 1993.

5. Gross PA, Hermogenes AW, Sacks HS, Levandowski RA. The efficacy of influenza vaccine in elderly persons. A meta-analysis and review of the literature. Annals of Internal Medicine 123:518-527, 1995.

6. Honkamen PO, Keistinen T, Kivelä SL. Reactions following administration of influenza vaccine alone or with pneumococcal vaccine to the elderly. Archives of Internal Medicine 156: 205208, 1996.

7. Margolis KL, Nichol KL, Poland GA, Pluhar RE. Frequency of adverse reactions to influenza vaccine in the elderly. Journal of American Medical Association 264:1139-1141, 1990.

8. Ministério da Saúde. Programa de Saúde do Idoso. disponível em <http://www.saude.gov.br/programas/idoso/programa.num>, Brasilia, 2001.

9. Nicholson KG, Kent J, Hammersley V, Cancio, E. Acute viral infections of upper respiratory tract in elderly people living in the community: comparative, prospective, population based study of disease burden. British Medical Journal 315:10601064, 1997.

10. Polland GA, Borrud A, Jacobson RM, Mcdermott K, Wollan PC, Brakke D, Charboneau JW. Determination of deltoid fat pad thickness - Implications for needle length in adult immunization. Journal of American Medical Association 277:1709-1711, 1997.

11. Rothman K, Greenland S. Modern Epidemiology, 2nd edition, Lippincott-Raven, New York, 1998.

12. Roy G, Fradet M, Le Hénaff $D$. Vaccination contre la grippe des personnes âgées: quelques préjuges courants. Revue Cannadienne de Santé Publique 87:298-300, 1996.

13. Rumel D. Indicadores de mortalidade por categorial ocupacional e nível social. Dissertação de Mestrado, Universidade de São Paulo, São Paulo, 1987.

14. Secretaria de Estado de Saúde de São Paulo (SES/SP). Campanha Nacional de Vacinação para o Idoso. Centro de Vigilância Epidemiológica, Informe Técnico. São Paulo, 2001.

15. Toniolo Neto J, Weckx LW, Halker E, Lopes CH, Succi RCM, Paiva TM, Ishida MA, Forleo Neto E. Safety of simultaneous pneumococcal and influenza vaccination in elderly patients in Brazil. Drugs Aging 15:43-45, 1999.

16. Weckx LY, Lopes $\mathrm{CH}$, Kobayashi FK. Avaliação da tolerância à vacina contra influenza e à vacina contra pneumococo em idosos. Boletim Informativo do Centro de Vigilância Epidemiológica - Secretaria Estadual de Saúde de São Paulo 14:7-10, 1999. 